\section{P746 MATERNAL TO CHILD TRANSMISSION PREVENTION PROGRAM FOR SYPHILIS AND HIV IN BRAZIL: MISSED OPPORTUNITIES}

${ }^{1}$ Angelica Miranda* ${ }^{2}$ Mariangela Silveira, ${ }^{3}$ Maria Alix Araujo, ${ }^{4}$ Leonor Lannoy, ${ }^{5}$ Sandra Moreira-Silva, ${ }^{6}$ Leila Silva, ${ }^{7}$ Adele Benzaken, ${ }^{8}$ Valeria Saraceni. ${ }^{1}$ Universidade Federal do Espirito Santo, Departamento de Medicina Social, Vitoria, Brazil; ${ }^{2}$ Universidade Federal de Pelotas, Pelotas, Brazil; ${ }^{3}$ Universidade Fortaleza, Fortaleza, Brazil; ${ }^{4}$ Secretaria de Saúde do Distrito Federal, Brasilia, Brazil; ${ }^{5}$ Secretaria de Saúde do Estado do Espírito Santo, Vitoria, Brazil; ${ }^{6}$ Secretaria de Estado de Saúde do Amazonas, Manaus, Brazil; " Ministerio da Saúde do Brasil, Brazil; ${ }^{8}$ Secretaria Municipal de Saúde do Rio de Janeiro, Rio de Janeiro, Brazil

\subsection{6/sextrans-2019-sti.805}

Background While antenatal screening for HIV and syphilis are part of the national policy in Brazil, screening and treatment coverage remain inadequate in many parts of the country. The goal of this study was to describe missed opportunities of mother-to-child transmission (MTCT) from the point of view of pregnant women, health professionals and health care managers.

Methods A semi-structured interview was conducted in six Brazilian States. Pregnant women, health professionals and unit managers were interviewed focusing on identifying failures in the process of pregnant women care and MTCT of syphilis or HIV. The project's approach was quantitative, but open-ended questions were included to capture the views of participants regarding feasibility of strategies being adopted for controlling MTCT.

Results A total of 109 women, 62 health professionals and 34 health care managers participated in the study. The median age of women was 24(range 15-46) years old and the median age of schooling was 8 years. Eighty-percent of those interviewed were enrolled in prenatal care. Among those who attend antenatal visits the median was 6.4(range 1-20) visits. Managers and health professionals had a median of 10(range 4-25) years of working. Less than 50\% of Health professionals and managers had been trained in HIV and syphilis MTCT; 79\% reported that they needed to receive more training. In the interviews the managers said they had provided tests and treatment for these infections, but health professionals said they did not had available tests or treatment to offer to and the women complaint about the difficulties to receive treatment. Women complained they were not prepared to talk about the diagnosed infections with their partner.

Conclusion It is a challenge to organize the logistics and breaking down barriers to care in Brazil. Health care system and policy factors can help to eliminate MTCT when they promote knowledge on strategies being adopted for the control of these infections.

Disclosure No significant relationships.

\section{P747 CHARACTERISTICS OF CHLAMYDIA/GONORRHEA INFECTIONS ASSOCIATED WITH A SUBSEQUENT SYPHILIS DIAGNOSIS IN BRITISH COLUMBIA, CANADA}

${ }^{1}$ Theodora Consolacion*, ${ }^{1}$ Janyn Mercado, ${ }^{1}$ Olga Mazo, ${ }^{1}$ Venessa Ryan, ${ }^{2}$ Linda Hoang, ${ }^{2}$ Muhammad Morshed, ${ }^{1}$ Mark Gilbert, ${ }^{3}$ Mark Hull, ${ }^{1}$ Troy Grennan, ${ }^{1}$ Jason Wong. 'British Columbia Centre for Disease Control, Vancouver, Canada; ${ }^{2}$ British Columbia Centre for Disease Control Public Health Laboratory, Vancouver, Canada; ${ }^{3}$ British Columbia Centre for Excellence in HIVIAIDS, Vancouver, Canada

10.1136/sextrans-2019-sti.806
Background Since 2010, infectious syphilis rates have risen dramatically in British Columbia (BC), Canada. We examined whether characteristics of a chlamydia (CT) or gonorrhea (GC) diagnosis were associated with a subsequent infectious syphilis diagnosis.

Methods All CT and GC diagnoses in BC from 2006 to 2017 were linked to infectious syphilis diagnoses in the subsequent 12-month period. A multivariable logistic regression model was used to identify factors associated with a subsequent infectious syphilis diagnosis and adjusted odds ratios (aOR) with 95\% confidence intervals (CI) were reported.

Results Of the 133,264 CT/GC diagnoses, 819 (0.6\%) linked to a subsequent syphilis diagnosis. Most were male $(777 / 819$, $94.9 \%$ ) with a mean age of 36.1 years (standard deviation $=11.2$ years) and had $\geq 3$ CT/GC diagnoses (419/819, 51.2\%). At time of CT/GC diagnosis, 222 (27.1\%) were living with HIV which increased to $245(30.0 \%)$ at the time of a syphilis diagnosis. The odds of a subsequent syphilis diagnosis were greater among men $(\mathrm{aOR}=12.2$, 95\% CI: 8.4-17.7); older age groups of $25-29$ years $(\mathrm{aOR}=1.5,95 \% \mathrm{CI}: 1.1-2.1)$, 30-39 years $(\mathrm{aOR}=2.4,95 \% \mathrm{CI}: 1.8-3.2), \quad 40-59$ years $(\mathrm{aOR}=3.7,95 \% \mathrm{CI}: 2.8-4.9)$, and $\geq 60$ years $(\mathrm{aOR}=2.6,95 \%$ CI: $1.3-5.0)$ when compared to age group 20-24 years; those living with $\mathrm{HIV}$ at time of CT/GC diagnosis $(\mathrm{aOR}=9.9,95 \%$ CI: 7.6-12.9); those with a history of lymphogranuloma venereum $(\mathrm{aOR}=3.4,95 \% \mathrm{CI}: 2.3-5.2)$; those with a CT/GC diagnosis from 2012 onward $(\mathrm{aOR}=4.7,95 \% \mathrm{CI}$ : 3.7-5.9); and those with a history of 3 or $4 \mathrm{CT} / \mathrm{GC}$ diagnoses $(\mathrm{aOR}=13.2$, 95\%CI: $10.6-16.3)$ or $5+\mathrm{CT} / \mathrm{GC}$ diagnoses $(\mathrm{aOR}=44.6$, 95\% CI: 34.8-57.1) when compared to those with 1 or 2 diagnoses.

Conclusion Characteristics of CT/GC diagnoses that were associated with a subsequent infectious syphilis diagnosis included male gender, older than age 24 years, co-infected with HIV, history of lymphogranuloma venereum, diagnosis of CT/GC from 2012 onward, and a history of 3+ CT/GC diagnoses. Disclosure No significant relationships.

\section{P748 SYPHILIS DIAGNOSTIC TEST OF STANDARDTM $Q$ SYPHILIS AB USING FINGERPRICK WHOLE BLOOD AND SERUM ON HIGH RISK POPULATIONS}

Rahma Lubis*, Sondang Sirait, Triana Agustin, Wresti Indriatmi. Faculty of Medicine Universitas Indonesia - Dr. Cipto Mangunkusumo National General Hospital, Dermatology and Venereology Department, Central Jakarta, Indonesia

\subsection{6/sextrans-2019-sti.807}

Background Syphilis is still a worldwide health problem with $80-90 \%$ of new cases occurring in developing countries with little or no diagnostic access. The availability of new diagnostic test such as rapid test or point-of-care test can improve the medical care of syphilis. Most rapid syphilis tests currently available are treponemal tests, one of them is STANDARD ${ }^{\mathrm{TM}}$ Q Syphilis Ab. This study aims to assess STANDARD ${ }^{\mathrm{TM}} \mathrm{Q}$ Syphilis Ab's rapid test capability using serum and fingerprick whole blood specimens compared with Treponema pallidum Haemagglutination Assay (TPHA) as the gold standard in detecting syphilis in high-risk populations comprised of transgenders, men who have sex with men, and female sex workers.

Methods This study is a diagnostic test with a cross sectional study design done in January 2018 in Pasar Rebo Public Health Center, East Jakarta, Indonesia. Samples were selected 
consecutively with total of 127 samples. All steps in this research; history taking, physical examination, and blood tests were done blindedly.

Results The results of this study using serum specimens were sensitivity of $91.30 \%$, specificity of $97.53 \%$, positive predictive value $95.45 \%$, negative predictive value of $95.18 \%$, and accuracy 95.28. Test results with fingerprick whole blood specimens gave sensitivity of $84.78 \%$, specificity of $98.77 \%$, positive predictive value of $97.50 \%$, negative predictive value of $91.95 \%$, and accuracy $93.70 \%$. Compatibility of rapid test STANDARD $^{\mathrm{TM}}$ Q Syphilis $\mathrm{Ab}$ results between serum and fingerprick whole blood specimens was very $\operatorname{good}(\kappa=0.8223)$.

Conclusion Rapid test STANDARD ${ }^{\mathrm{TM}}$ Q Syphilis Ab can be used as an option for treponemal test in supporting syphilis diagnosis, either as routine screening or confirmation of nontreponemal test result. The fingerprick whole blood specimen can be used as treponemal test alternative which is faster and easier to do.

Disclosure No significant relationships.

\section{P750 A COMPARISON OF TREPONEMA PALLIDUM MOLECULAR TYPING SYSTEMS: MLST VS. ECDCT}

${ }^{1}$ Sharon Sahi*, 'Lauren Tantalo, ${ }^{2}$ Christina Marra. 'University of Washington, Neurology, Seattle, USA; ${ }^{2}$ University of Washington, USA

\subsection{6/sextrans-2019-sti.808}

Background Several syphilis typing systems have been proposed. Recent work suggests that multilocus sequence typing (MLST) may be superior to enhanced CDC typing (ECDCT), particularly because ECDCT type may differ among organisms amplified from different anatomical sites in the same person. The goal of this study was to compare the two systems.

Methods DNA was extracted from 20 Treponema pallidum isolates propagated in rabbits, 10 oral and 10 genital or non-genital lesion swabs, and 10 blood samples from patients with syphilis. MLST type for tp0136, tp0548 and tp0705 and ECDCT type were determined according to published methods. Samples were chosen because they were completely typeable by ECDCT. ECDCT types were also determined for samples from different anatomical sites in 7 patients, and from blood and blood isolates (rabbit propagated) in 8 patients.

Results MLST type could be fully determined for 19 (95\%) of 20 bacterial isolates, $8(80 \%)$ of 10 bloods, $7(70 \%)$ of 10 lesion swabs, and $5(50 \%)$ of 10 oral swabs. 13 subtypes were identified by ECDCT, and 12 by MLST. While MLST was able to subdivide two common ECDCT types (1.1.1, 1.1.2, 1.1.9, and 1.37 .1 within $14 \mathrm{~d} / \mathrm{f}$; and $1.3 .1,1.38 .1$, and 6.3.1 within $14 \mathrm{~d} / \mathrm{g}$ ), it failed to distinguish among less common ECDCT types. ECDCT type was identical in 5 paired lesion and oral swabs, 1 paired blood and oral swab, and 1 paired blood, lesion and oral swabs. In addition, ECDCT type was identical in 8 paired blood and blood isolates.

Conclusion Compared to ECDCT, determination of MLST was less often successful from isolates and from clinical samples, and it was not uniformly more discriminating. ECDCT was stable among anatomical sites and between direct patientderived samples compared to rabbit propagated organisms.

Disclosure No significant relationships.

\section{P751 EVALUATION OF A SYPHILIS AWARENESS CAMPAIGN FOR GAY, BISEXUAL AND OTHER MSM (GBMSM): DID WE REACH OUR TARGETED AUDIENCE?}

${ }^{1}$ Jason Wong*, ${ }^{2}$ Shenyi Pan, ${ }^{1} E m m a$ Cumming, ${ }^{2}$ Heather Armstrong, ${ }^{2}$ Nicanor Bacani, ${ }^{1}$ Devon Haag, ${ }^{3}$ Venessa Ryan, ${ }^{1}$ Jillian Arkles Schwandt, ${ }^{1}$ Troy Grennan, ${ }^{4}$ Jody Jollimore, ${ }^{5}$ Nathan Lachowsky, ${ }^{2}$ David Moore. ${ }^{1} B C$ Centre for Disease Control, Clinical Prevention Services, Vancouver, Canada; ${ }^{2} B C$ Centre for Excellence in HIVIAIDS, Epidemiology and Population Health, Vancouver, Canada; ${ }^{3}$ British Columbia Centre for Disease Control, Clinical Prevention Services, Vancouver, Canada; ${ }^{4}$ Community Based Research Centre, Vancouver, Canada; ${ }^{5}$ University of Victoria, School of Public Health and Social Policy, Victoria, Canada

\subsection{6/sextrans-2019-sti.809}

Background In 2017, we launched a syphilis awareness campaign ("Syphistory") targeted towards gay, bisexual, and other men who have sex with men (gbMSM). Using data from a study of gbMSM in Vancouver, we describe participants who reported seeing Syphistory and whether it reached gbMSM at higher risk of syphilis.

Methods Participants aged $\geq 16$ years who reported having sex with another man in the previous six months were recruited through respondent-driven sampling. We analyzed data collected from September 17, 2017 to August 31, 2018. Characteristics of participants who reported seeing the campaign were compared using Wilcoxon rank-sum and chi-square/Fisher's exact test. Multivariate logistic regression was used to examine the association between seeing Syphistory and recently being tested for syphilis, controlling for potential confounding factors, namely age, HIV status, place of residence, education level, and recent illicit drug use (IDU).

Results Of the 383 participants who responded, 103 (27\%) reported seeing Syphistory. Participants who saw Syphistory tended to be younger (median 30 vs 32 years old, $\mathrm{p}=0.03$ ), live in downtown Vancouver $(58 \%$ vs $38 \%, \mathrm{p}=0.46)$, had greater than a high school education (97\% vs $89 \%, \mathrm{p}=0.08)$, and were known to be HIV-negative ( $92 \%$ vs $81 \%, \mathrm{p}=0.15$ ). They reported more male sex partners (median 4 vs 3 , $\mathrm{p}=0.12)$, condomless anal sex $(92 \%$ vs $81 \%, \mathrm{p}=0.06)$, and IDU $(86 \%$ vs $72 \%, \mathrm{p}=0.04)$ in the last 6 months. Almost $75 \%(52 / 86)$ of those who saw Syphistory reported a syphilis test within the last 3 months compared with 58\% (97/244) who did not see Syphistory $(p=0.01)$. Participants who saw Syphistory had a greater odds $(\mathrm{aOR}=3.63$; 95\% CI, 1.28 10.27) of a syphilis test within 3 months, versus no or unknown previous syphilis test.

Conclusion Participants who saw Syphistory tended to report behaviours that may increase the risk of syphilis infection and were more likely to have had a recent syphilis test.

Disclosure No significant relationships.

\section{P752 UNDERSENSITIVE NON-TREPONEMAL TESTS: IMPLICATIONS FOR SYPHILIS MANAGEMENT}

John Scythes*, Colman Jones. Community Initiative for AIDS Research, Toronto, Canada

\subsection{6/sextrans-2019-sti.810}

Background Syphilis management has traditionally been based on non-treponemal tests (NTT). However, investigations over 3 decades have revealed serious issues with the sensitivity of NTT. 